\title{
On the Benefits of Head Stabilization with a View to Control Balance and Locomotion in Humanoids
}

\author{
Ildar Farkhatdinov and Vincent Hayward \\ UPMC Univ Paris 06, UMR 7222, \\ Institut des Systèmes Intelligents et de Robotique \\ Paris, France \\ Email: \{ildar, hayward\}@isir.upmc.fr
}

\author{
Alain Berthoz \\ Laboratoire de Physiologie de la Perception et de l'Action, \\ Collège de France \\ Paris, France \\ Email: alain.berthoz@ college-de-france.fr
}

\begin{abstract}
The estimation of the gravitational vertical is a fundamental problem faced by locomoting robots and animals alike. We describe a technique to address this problem that involves a damped inclinometer, an inertial measurement unit mounted on an actuated orienting platform, that is a robot head, so-to-speak. Simulations show that a nonlinear observer based on Newton's method to solve the full dynamics of the system given inertial sensor data gives accurate verticality estimates even in the presence of highly dynamic perturbations that include large fictitious force terms. Moreover, when the sensor platform is servoed to the estimate of the gravitational vertical to provide for horizontal stabilization, the accuracy of the estimate is improved by almost two orders of magnitude. Similar gains of performance are observed even in the presence of noise and parameter uncertainty.
\end{abstract}

\section{INTRODUCTION}

Locomotion is the raison d'être of humanoid robots. Humans, for their part, rely on a multiplicity of sensory inputs and sophisticated anticipatory mechanisms to solve the control problems subserving standing, walking, running, jumping, dancing, and so on. Vestibular inputs play a central role in all these tasks, which are achieved through a combination of postural movements and forces and torques exerted against the environment. These tasks become exceedingly difficult, or even impossible, when the vestibular system is impaired, even in the presence of the other sensory inputs.

In robotic devices, the counterpart of the vestibular system plays is the so-called 'inertial measurement unit' (or IMU in short) that combines accelerometers and rate-gyros. Accelerometers are often utilized as tilt sensors and gyroscopes are used to measure the change in the robot's orientation. A common design wisdom wants that the IMU be located close to the center of mass of a humanoid robot. In [1], [2], [3] and [4], accelerometers and gyroscopes were placed in the humanoid's torso; in [5] and [6], an IMU was placed in the pelvis; and in [7] an IMU was attached to the hip of the robot. Few humanoid robots had an IMU located in the head. The sole example that we could find is described in [8].

In humans, the head-located vestibular system is known to participate in a number of functions that include gaze stabilization through the vestibular-ocular reflex and the sense of spatial orientation, but that also makes it possible to create a ground-independent inertial frame from which postural control can be more effectively performed. It has been proposed by Pozzo and Berthoz [9], [10], [11], that during locomotion the head is stabilized in rotation by using the vestibular inertial information This provides the brain with a mobile reference frame, which, in cooperation with vision and gaze allows a 'top-down' control of locomotion.

Here, we propose to illuminate the advantages of locating an IMU in a the head of a humanoid, rather than in any other parts of its anatomy. The knowledge of the direction of the gravity vector, that is of the gravitational vertical, is essential to achieve stance and locomotion since the gravitational vertical may be poorly estimated from visual cues or from the relationship of the robot to the ground.

Locomoting animals share the behavioral trait of maintaining a stable head, hence a stable vestibular system, during locomotion. This behavior is discussed in the next section. A stabilized robotic head would presumably benefit from the same advantages as those of natural heads and these observations led us to believe that humanoid robots should also adopt a similar strategy. Among numerous other potential advantages, it is supposed that a robot head that is horizontally stabilized during locomotion facilitates the estimation of the gravitational vertical.

In this paper, we consider the problem of gravitational vertical estimation with consideration of the nonlinear dynamics of an inclinometer combined with an IMU, and we show that the horizontal stabilization of a robot's head yields a dramatic improvement in the estimation of the gravitational vertical in the face of strong perturbations, compared to when the same sensors are rigidly attached to an arbitrary body of a humanoid's kinematic tree.

\section{Previous Works in Verticality Estimation}

Generally speaking, inertial sensors are noisy because they pick-up vibrations that become added to the low frequency components of interest of the acceleration and velocity signals. Gyroscopic measurements also suffer from bias and are highly sensitive to dynamic errors.

To combat these problems, approaches to the design state observers and sensor fusion methods have been proposed to improve inertial measurements [12]. For instance, in [13], a Kalman filter was used to estimate the vertical direction 
from the tilt measurements in the linearized, planar case. Kalman filters were also applied to the attitude estimation of accelerated rigid bodies in three dimensions from the fused measurements of gyroscopes and accelerometers [14]. In [15], a nonlinear observer for attitude estimation based on gyroscope measurements is described, but only kinematic relationships were considered in the model. A similar problem was solved in [16], where a nonlinear observer combined inertial and visual information. None of these works considered the full system dynamics.

\section{GRaVitational Verticality in Humans}

Behavioral studies have shown that humans stabilize their heads in rotation for different locomotor tasks, such as free walking, walking in place, running in place and hopping [9], [10]. This stabilization probably uses a cooperation between both the measurement of head rotations by the semi-circular canals and the measure of translations by the utriculus and sacculus (otolith organs). The plane of the stabilization is determined by the task; it can vary and be controlled by gaze. Further experiments showed that total darkness did not significantly influence the stabilization of the head, which demonstrated the importance of this behavior in the coordination of the multiple degrees of freedom of the body during gait. Later, it was shown that head stabilization occurred also in the frontal plane during the maintenance of monopodal and bipodal equilibrium on unstable rocking platforms [11]. The head remained stable relatively to the vertical, despite large translations in the frontal plane. Head angular stabilization was essential for effective postural control during complex equilibrium tasks. Additional studies suggest that the motion of the head, together with gaze control, is closely related to postural control during locomotion [17].

At the neural level, it is also believed that the estimation of the vertical direction from various sensor inputs is a fundamental brain function where putative computational tasks and their neural correlates have been studied in humans and animals models [18], [19], [20].

\section{A Robotic Sense of Gravitational Verticality}

In order to emulate some aspects of the function of the vestibular system, we used the gravity-referenced inclinometer (Model A900 from Applied Geomechanics, see Fig. 1) as the primary sensor to measure orientation with respect to the gravity vector. The sensing element is a glass vial partially filled with a conductive liquid. When the sensor is level, the four internal electrodes are immersed in the liquid at equal depths. When the sensor tilts, the depth of immersion of the electrodes changes, altering the electrical resistance between matched pairs of electrodes. Figure 1, left panel, shows this liquid-based inclinometer in planar side view. A damped pendulum, Fig. 1 right panel, can be used as a simplified mechanical model of the sensor.

We selected a liquid-based inclinometer as a tilt sensor, because it has several important benefits compared to the
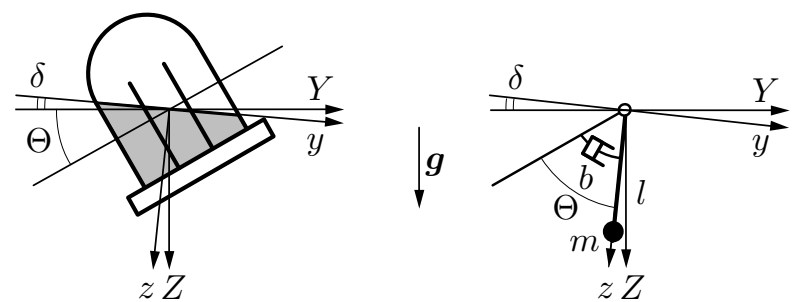

Fig. 1. Inclinometer (Model A900 from Applied Geomechanics). Side view of the liquid half-filled vial and damped pendulum as a model. Its measurements are affected by the 'fictitious forces', if the sensor frame is not inertial. Here, the inclinometer is accelerated to the right. The same error could be caused by an angular movement.

accelerometers that are often used as tilt sensors. Most importantly, inclinometers provide quasi-linear tilt measurements, while accelerometers necessarily provide measurements that are nonlinearly related to the gravitational vertical.

Another disadvantage, already alluded to, is that accelerometers are highly sensitive to vibrations and can be used as tilt sensors in the low frequencies only. Finally, due to their mechanical structure liquid-based dual-axis inclinometers provides tilt measurements independent, around two orthogonal axes that can be directly mapped to rotation matrix defining the orientation of the inclinometer with respect to the gravity vector.

We would like to suggest that these three characteristics are in fact shared with natural vestibular systems, which are also linearized, naturally low-passed, and decoupled [21]. In this sense, the liquid-based inclinometer possesses intriguing biomimetic characteristics.

A simple robotic head could comprise a two degree-offreedom, actuated pointing mechanism. A local coordinate frame, $\{x, y, z\}$, is attached to the surface of the fluid inside the sensor. Another coordinate frame, $\{X, Y, Z\}$, shares the same origin, but remains aligned with the gravity vector, $\boldsymbol{g}$. If the latter frame is not inertial, the two frames differ due to presence of relative acceleration, centrifugal, Coriolis and Euler forces that will act as forcing terms on the dynamics of the sensor, causing the measurement to be unreliable.

This problem can be visualized in the plane, as illustrated in Fig. 1. The figure shows the inclinometer being accelerated to the right, or to be under the influence of an angular movement. In three dimensions, the four different 'fictitious forces' mentioned in the previous paragraph could in fact have the same effect on the sensor, necessitating the design of a dynamic observer to resolve the ambiguity. This question must be addressed in three dimensions, which is the subject of the next sections.

Before doing so, we propose to model the inclinometer as a three-dimensional damped pendulum with concentrated mass $m$, viscous damping $b$ and length $l$ shown in projection in Fig. 1, and where the $x$ component of the measurement error, $\delta$, is also represented. The pivot of the pendulum undergoes linear accelerations and the attachment of the damper is rotated. 
We suppose that the inclinometer is mounted on an orientable platform, modeled here as an actuated gimbal mechanism attached to the rest of the robot. Two actuators provide two torques, that act through the kinematics of the gimbal to provide a torque, $\boldsymbol{\tau}=\left(\begin{array}{ll}\tau_{x} & \tau_{y}\end{array}\right)^{\top}$ acting on the angular movements of the platform. Lastly, the platform also has an accelerometer that measures the acceleration of the origin the local frame and rate-gyros measuring its angular velocity.

\section{Observation of The Gravitational Vertical}

\section{A. Modeling}

We first consider the case when the inclinometer is attached to the robot's body (Fig. 2a). The inclinometer and the IMU rotate and translate together with the torso, which is the conventional practice for most modern humanoid robots. For purposes of comparison, we rather consider that the sensing platform is attached to the head of the robot but that the head is rigidly attached to the main body of the robot.
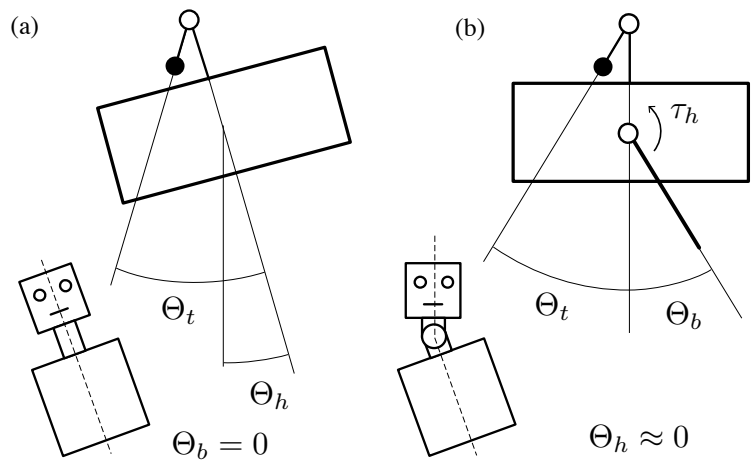

Fig. 2. Planar representation. (a) The sensing platform is fixed to a moving robot body. (b) The sensing platform is attached to a stabilized head.

The full unforced dynamic model of the damped spherical pendulum oscillating in three dimensions was given in reference [22],

$$
\begin{aligned}
\boldsymbol{J}(\dot{\boldsymbol{\varphi}}-\dot{\boldsymbol{\omega}}) & =m \boldsymbol{g} \boldsymbol{\rho} \times \boldsymbol{R}^{\top} \boldsymbol{e}_{3}+m \boldsymbol{\rho} \times \boldsymbol{R}^{\top} \boldsymbol{a}+b \boldsymbol{\omega}, \\
\dot{\boldsymbol{R}} & =\boldsymbol{R}(\hat{\boldsymbol{\varphi}}-\hat{\boldsymbol{\omega}}),
\end{aligned}
$$

where the angular velocities of the pendulum and of the platform are $\boldsymbol{\omega}=\left(\begin{array}{lll}\omega_{x} & \omega_{y} & 0\end{array}\right)^{\top}$ and $\boldsymbol{\varphi}=\left(\begin{array}{lll}\varphi_{x} & \varphi_{y} & 0\end{array}\right)^{\top}$, respectively, since rotations around the third axis are not permitted. The pendulum tensor of inertia in the body-fixed frame is $\boldsymbol{J}$. The other parameters include $m$ and $b$, the mass and damping of the pendulum, respectively, $\boldsymbol{g}$, the gravity vector, $\boldsymbol{a}$, the acceleration of the pivot point, and $\boldsymbol{e}_{3}=\left(\begin{array}{lll}0 & 0 & 1\end{array}\right)^{\top}$, a unit vector along $z$. An angular velocity, $\omega$, corresponds to a skew-symmetric matrix, $\hat{\boldsymbol{\omega}}$. The rotation matrix, $\boldsymbol{R} \in S O(3)$, describes the orientation of pendulum and $\boldsymbol{\rho}=\left(\begin{array}{lll}0 & 0 & l\end{array}\right)^{\top}$, the position of the center of mass. The matrix, $R$, can be expressed in terms of the projections of the pendulum's local frame unit vectors to the frame $\{X, Y, Z\}$,

$$
\boldsymbol{R}=\left(\begin{array}{lll}
{ }^{x} r_{X} & { }^{y} r_{X} & { }^{z} r_{X} \\
{ }^{x} r_{Y} & { }^{y} r_{Y} & { }^{z} r_{Y} \\
{ }^{x} r_{Z} & { }^{y} r_{Z} & { }^{z} r_{Z}
\end{array}\right),
$$

where ${ }^{\lambda} r_{\Lambda}$ stands for the projection of a unit vector $\lambda$ onto an axis $\Lambda$. The measurements, $\Theta_{x}$ and $\Theta_{y}$, from the inclinometer relate to the elements of matrix $\boldsymbol{R}$ as follows (among possibilities),

$$
\Theta_{x}=\arctan \frac{{ }^{y} r_{Z}}{{ }^{z} r_{Z}}, \quad \Theta_{y}=\arctan \frac{{ }^{x} r_{Z}}{{ } r_{Z}} .
$$

Next, consider the combined dynamics of the pendulum and of the head, forced by the torque, $\tau$,

$$
\begin{aligned}
\boldsymbol{J}(\dot{\boldsymbol{\varphi}}-\dot{\boldsymbol{\omega}}) & =m \boldsymbol{g} \boldsymbol{\rho} \times \boldsymbol{R}^{\top} e_{3}+m \boldsymbol{\rho} \times \boldsymbol{R}^{\top} \boldsymbol{a}+b \boldsymbol{\omega}+\not / \\
\dot{\boldsymbol{R}} & =\boldsymbol{R}(\hat{\boldsymbol{\varphi}}-\hat{\boldsymbol{\omega}}), \\
\boldsymbol{K} \dot{\varphi} & =\boldsymbol{\tau}, \\
\dot{\boldsymbol{P}} & =\boldsymbol{P} \hat{\boldsymbol{\varphi}}
\end{aligned}
$$

where $\boldsymbol{P}$ is the rotation matrix describing the frame of the platform, $\boldsymbol{K}$, the inertia tensor of the platform in a bodyfixed frame, and $\tau$ the control torque applied to it. After some algebra, the two systems can be put in state-space form; first the fixed, unactuated head system,

$$
\begin{aligned}
\dot{\boldsymbol{\omega}} & =-\boldsymbol{J}^{-1}\left(m \boldsymbol{g} \boldsymbol{\rho} \times \boldsymbol{R}^{\top} \boldsymbol{e}_{3}+m \boldsymbol{\rho} \times \boldsymbol{R}^{\top} \boldsymbol{a}+b \boldsymbol{\omega}\right), \\
\dot{\boldsymbol{R}} & =\boldsymbol{R}(\hat{\boldsymbol{\varphi}}-\hat{\boldsymbol{\omega}}),
\end{aligned}
$$

and then the actuated head system,

$$
\begin{aligned}
\dot{\boldsymbol{\omega}}_{p} & =\boldsymbol{K}^{-1} \boldsymbol{\tau}-\boldsymbol{J}^{-1}\left(m \boldsymbol{g} \boldsymbol{\rho} \times \boldsymbol{R}^{\top} \boldsymbol{e}_{3}+m \boldsymbol{\rho} \times \boldsymbol{R}^{\top} \boldsymbol{a}+b \boldsymbol{\omega}\right), \\
\dot{\boldsymbol{R}} & =\boldsymbol{R}(\hat{\boldsymbol{\varphi}}-\hat{\boldsymbol{\omega}}), \\
\dot{\boldsymbol{\varphi}} & =\boldsymbol{K}^{-1} \boldsymbol{\tau}, \\
\dot{\boldsymbol{P}} & =\boldsymbol{P} \hat{\boldsymbol{\varphi}}
\end{aligned}
$$

The system state, $\boldsymbol{x}=\left(\begin{array}{llll}\boldsymbol{\omega} & \boldsymbol{R} & \boldsymbol{\varphi} & \boldsymbol{P}\end{array}\right)^{\top}$ is, by abuse of notation, made of the elements of the vectors and the matrices are arranged in a single vector from the first to the last. The state has 24 components. We can now represent the systems by expressions of the form

$$
\dot{\boldsymbol{x}}=F(\boldsymbol{x}, \boldsymbol{u}), \boldsymbol{y}=H(\boldsymbol{x}),
$$

where $F$ defines the flow of the nonlinear dynamics and $\boldsymbol{u}=$ $\left(\begin{array}{ll}a & \tau\end{array}\right)^{\top}$ is an input imposed by the movements of the robot combined with the torque applied to the head. The output, $\boldsymbol{y}$, is a function of projections from inclinometer's rotation matrix. To stabilize the head horizontally, it suffices to apply a PD control since it is a simple articulated mechanism, that is,

$$
\boldsymbol{\tau}=K_{p}(I-\boldsymbol{R})+K_{d}(-\dot{\boldsymbol{R}}),
$$

which servoes the head orientation to the gravitational vertical, provided that $\boldsymbol{R}$ be estimated by means of an observer, since it cannot be measured.

\section{B. Newton observer overview}

We recall the principle of nonlinear observers based on the Newton's method which rely on the numerical solution of the discretized system equations, in a dead-beat fashion, from the knowledge of past inputs and outputs [23]. These observers apply to large classes of smooth nonlinear systems such as our 
robotic head-vestibular system. The system is sampled with period $T$ to give,

$$
\boldsymbol{x}_{k+1}=F_{T}\left(\boldsymbol{x}_{k}, \boldsymbol{u}_{k}\right), \boldsymbol{y}_{k}=H\left(\boldsymbol{x}_{k}, \boldsymbol{u}_{k}\right),
$$

and the history of measurements and control inputs for a given time window, $N$, is

$$
\begin{aligned}
& \boldsymbol{Y}_{k} \triangleq\left(\begin{array}{llll}
\boldsymbol{y}_{k-N+1} & \boldsymbol{y}_{k-N+2} & \cdots & \boldsymbol{y}_{k}
\end{array}\right)^{\top}, \\
& \boldsymbol{U}_{k} \triangleq\left(\begin{array}{llll}
\boldsymbol{u}_{k-N+1} & \boldsymbol{u}_{k-N+2} & \cdots & \boldsymbol{u}_{k}
\end{array}\right)^{\top} .
\end{aligned}
$$

From the system model, we can express the state given the information provided by the last $N$ steps,

$H_{T}\left(\boldsymbol{x}_{k-N+1}, \boldsymbol{U}_{k}\right) \triangleq\left(\begin{array}{c}h\left(\boldsymbol{x}_{k-N+1}\right) \\ h \circ F_{T}^{\boldsymbol{u}_{k-N+1}}\left(\boldsymbol{x}_{k-N+1}\right) \\ \vdots \\ h \circ F_{T}^{\boldsymbol{u}_{k-1}} \circ \ldots \circ F_{T}^{\boldsymbol{u}_{k-N+1}}\left(\boldsymbol{x}_{k-N+1}\right)\end{array}\right)$

an expression that is termed the 'observability mapping'.

The observer problem consists of solving $N$ nonlinear equations,

$$
\boldsymbol{Y}_{k}-H_{T}\left(\boldsymbol{x}_{k-N+1}, \boldsymbol{U}_{k}\right)=0,
$$

something that be done using Newton's iterative method,

$$
\boldsymbol{\xi}_{k}^{i+1}=\boldsymbol{\xi}_{k}^{i}+\left[\frac{\partial H_{T}}{\partial \boldsymbol{\xi}}\left(\boldsymbol{\xi}_{k}^{i}, \boldsymbol{U}_{k}\right)\right]^{-1}\left(\boldsymbol{Y}_{k}-H_{T}\left(\xi_{k}^{i}, \boldsymbol{U}_{k}\right)\right)
$$

for $i=0, \ldots, d-1$ with $d$ selected to give a desired accuracy. Going back in time, the estimated state can be reconstructed recursively,

$$
\hat{\boldsymbol{x}}_{k}=F_{T}^{\boldsymbol{u}_{k-1}} \circ \cdots \circ F_{T}^{\boldsymbol{u}_{k-N+1}}\left(\boldsymbol{\xi}_{k}^{d}\right) .
$$

\section{Newton Observer for Gravitional Verticality Estimation}

The system (1) is discretized,

$$
\begin{gathered}
\boldsymbol{x}_{k+1}=F\left(\boldsymbol{x}_{k}, \boldsymbol{u}_{k}\right) T+\boldsymbol{x}_{k} \triangleq F_{T}^{\boldsymbol{u}_{k}}\left(\boldsymbol{x}_{k}\right) \\
y_{1, k}=h_{1}\left(x_{k}\right) \triangleq \arctan \left(\frac{p_{1,3}}{p_{3,3}}\right)_{k}, \\
y_{2, k}=h_{2}\left(x_{k}\right) \triangleq \arctan \left(\frac{p_{1,2}}{p_{3,3}}\right)_{k}
\end{gathered}
$$

where the $p_{i, j}$ are the elements of the matrix $\boldsymbol{P}$. The outputs, $y_{1}$ and $y_{2}$, correspond to measurements, $\Theta_{y}$ and $\Theta_{x}$, from the inclinometer. We construct an observer to estimate each output independently. Here we show the observer design for estimating $\Theta_{y}$. The design for $\Theta_{x}$ is analogous.

From the governing equations of the actuated orienting platform written in Section V-A, we could first obtain a closed-form expression for the observability mapping of Section $\mathrm{V}-\mathrm{B}$, and then compute its inverse Jacobian matrix for the Newton iterations. Obtaining a closed-form solution would be extremely tedious. Instead we used a finite difference approximation of the Jacobian matrix by recalculating $H_{T}$ at each period.

At time $k$, the histories of tilt measurements $y_{1, k}$ and control inputs $\boldsymbol{u}_{k}=\left(\boldsymbol{a}_{k}, \boldsymbol{\tau}_{k}\right)^{T}$ are kept in a queue for the last 24 iterations. After $d$ Newton iterations, the estimate for $\boldsymbol{x}_{k-N+1}$ is forwarded in time by $N-1$ steps to obtain the estimate of the current state $\hat{\boldsymbol{x}}_{k}$ as shown at the end of Section V-B. In the most general case, the state has 24 components. The number of states can be reduced by excluding the angular velocities about the vertical axis and the redundant components of the rotation matrices.

\section{RESUlts}

We proceeded with simulations in order to evaluate the performance of our gravitational verticality observer. For these simulations, we picked the parameters to have some reasonable values, $m=50 \mathrm{~g}, l=0.1 \mathrm{~m}, \boldsymbol{J}_{x}=\boldsymbol{J}_{y}=m l^{2}, \boldsymbol{K}=100 \boldsymbol{J}$, $b=0.005 \mathrm{~N} \cdot \mathrm{ms}$. The sampling period was $1 \mathrm{~ms}$. The head orientation was controlled by a nicely tuned PD control ( $K_{p}=230 \mathrm{~N} / \mathrm{rad}$ and $K_{d}=15 \mathrm{Ns} / \mathrm{rad}$ ).

We applied the observer just described to the unactuated, fixed platform system and compared the results to when the same observer was applied to the actuated, servo-controlled platform with horizontal stabilization. The Newton iterations were run every $25 \mathrm{~ms}$ with $d=5$.

The tests involved driving the platform through a dynamic trajectory, $[x(t), y(t), \Theta(t)]=$ $0.25[\sin (0.5 \pi t), \sin (\pi t), \pi \sin (2 \pi t)]$, where $t$ is time, forming the Lissajous figure represented in Fig. 3. The history of the acceleration components is in the bottom panel.

The tests were performed under two conditions. In the first, an accurate model of the system dynamics was assumed. In the second, the systems parameters given to the observer differed by $10 \%$ from the actual value. In addition noise was added to the output, simulating quantization of the sensor signal.

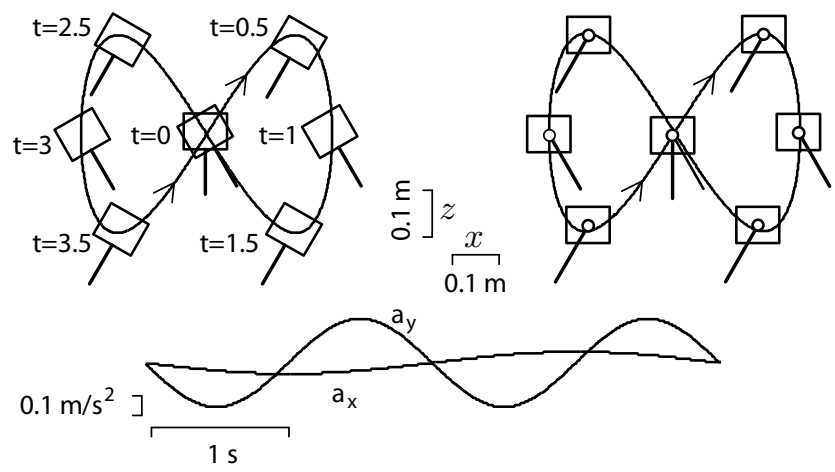

Fig. 3. Test trajectory used for comparison. In the left panel, the platform oscillates. In the right panel the platform is stabilized despite the movements of the body.

Figure 4 shows the simulation results for the fixed-platform system. It can be seen how the platform movements influenced the orientation of the inclinometer, causing the measurement to lag in phase. The observer, however, manages to estimate of the orientation of the platform reasonably well, that is with an error reaching $3^{\circ}$.

Figure 5 shows the results when the inclinometer was on a horizontally stabilized platform for the same trajectory. The platform orientation was almost zero, but the inclinometer oscillated with a magnitude of about $4^{\circ}$ due to the residual 


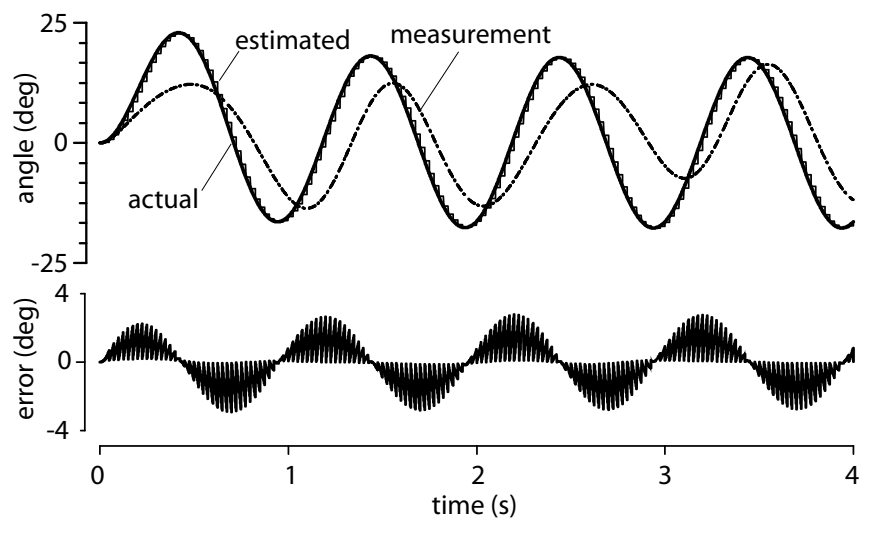

Fig. 4. Simulation with exact model when the platform is not stabilized (head).

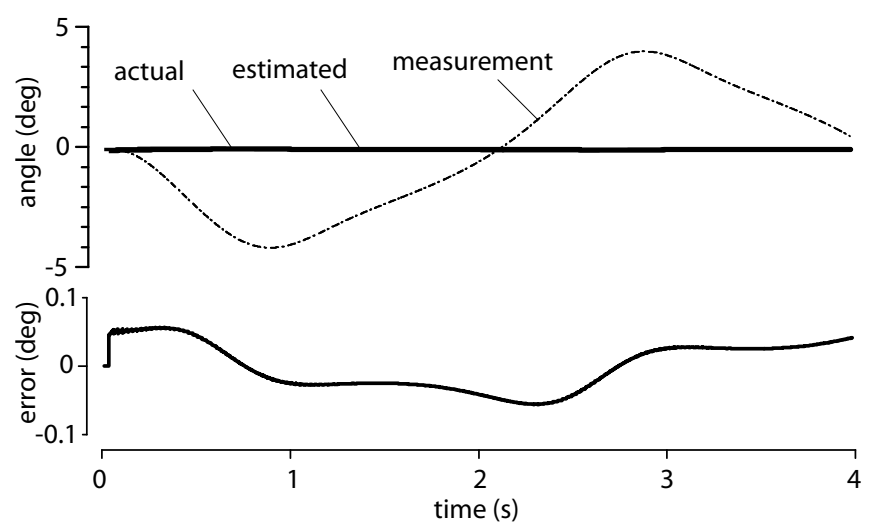

Fig. 5. Simulation with exact model information and stabilized platform (head).

acceleration. The estimation error with respect to the gravitational vertical was down to less that $0.1^{\circ}$.

Simulations were then performed when the model parameters given to the observer were over estimated by $10 \%$ : $\hat{m}=0.055 \mathrm{~kg}, \hat{l}=0.1 \mathrm{~m}, \hat{\boldsymbol{J}}=\hat{m} \hat{l}^{2}, \hat{\boldsymbol{K}}=100 \hat{\boldsymbol{J}}$, $\hat{b}_{t}=0.0055 \mathrm{~N} \cdot \mathrm{ms}$. In addition white Gaussian noise was added to the tilt measurement which corresponded to quantization error of a 16-bit analog-to-digital converter.

The results can be seen in Fig. 6 and in Fig. 7 for the fixed and actuated systems, respectively. The estimation error reached about $6^{\circ}$ for the fixed platform system, but when the horizontally stabilized platform was used, the estimation error never exceeded $0.5^{\circ}$.

\section{DisCUSSION AND CONCLUSION}

We set out to design a gravitational verticality estimation system that shares some features with the natural vestibular systems. Chiefly among them is the use of a (liquid) pendulum-type inclinometer that may be compared, in function, to the otolith organs of the natural vestibular systems in the sense that its measurements are naturally low passed. Like all gravitational and inertial sensors, an inclinometer is unable to make a distinction between the four terms of the gravito-inertial forces that can deviate it from its equilibrium.

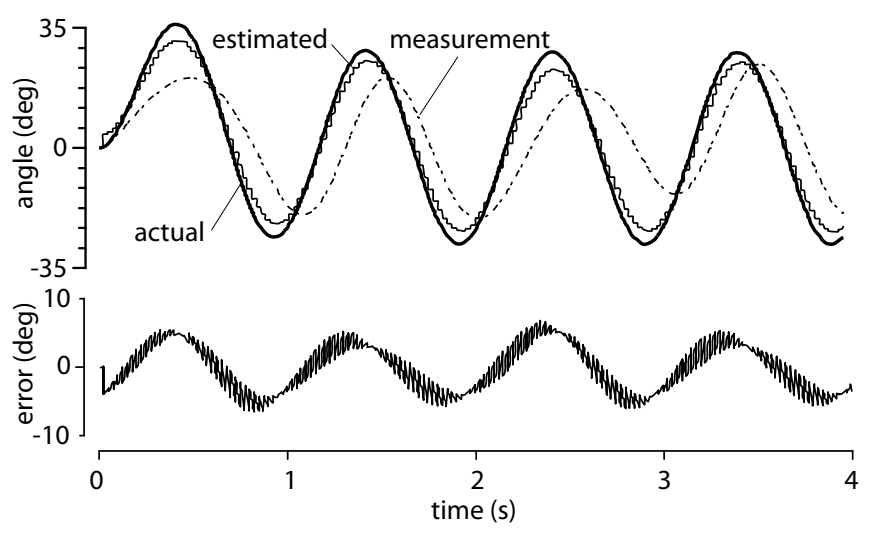

Fig. 6. Simulation with approximate model and noisy sensors when the platform (head) is not stabilized.

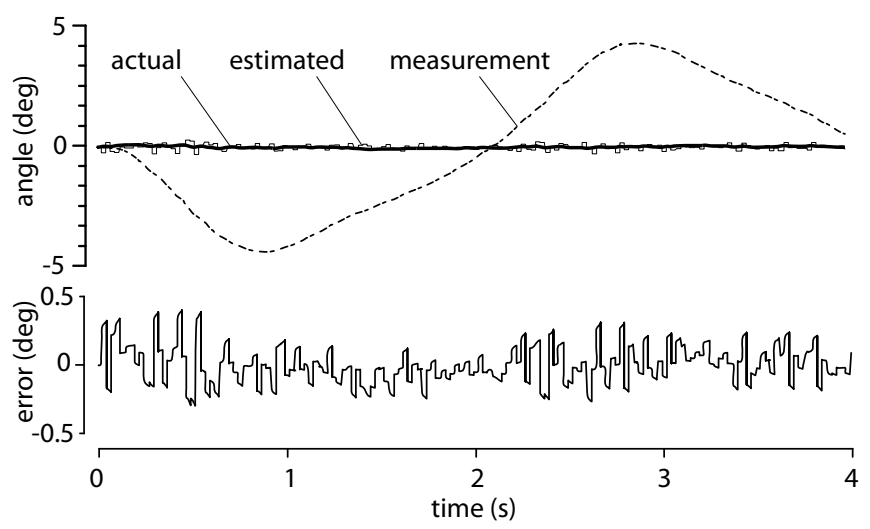

Fig. 7. Simulation with approximate model and noisy sensors with stabilized platform (head).

Using an IMU to provide additional acceleration and angular rate measurements, we showed that the gravitational component could be extracted almost exactly under highly dynamic trajectories, up to the accuracy of a nonlinear observer (using Newton iterations to solve the full system dynamics), even with uncertainty in the model parameters and noise in the measurements.

The second important feature that our system shares with many natural vestibular system is to be attached to an actuated, orientable platform able to stabilize the inertial measurement system in the horizontal plane, independently from the movements of the body to which it may be attached - a robot "head" so-to-speak. Slaving this platform to the output of the verticality observer could reduce the observer error by more than one order of magnitude under highly dynamic forcing trajectories. This observer may be compared in the function with the neural machinery associated with natural vestibular systems.

When the "head" of the robot was horizontally stabilized, then its angular velocity was zero, $\boldsymbol{\omega}=0$, and the rotation matrix, $\boldsymbol{R}$, that relates the measurements to the true vertical was close to identity, which simplified the dynamics of the system. In addition, it was shown that head stabilization was useful even if an exact model of the system was not known. 
It was also found that gains in accuracy were obtained by the same order as when the knowledge of an exact model was assumed.

Further simulation studies (not reported here) showed that in the case of stabilized head system to the reference given by the Newton's method based observer gave an absolute error smaller than $3^{\circ}$ when the dynamic parameter uncertainties reached up to $50 \%$. Conversely, the observer diverged when the head was not stabilized if the dynamic uncertainties exceeded $15 \%$. This means that head stabilization strategy considerably enlarges the convergence area of the observer and makes it more robust to model with respect to model uncertainties.

Since the invention of the marine compass, stabilized inertial platforms have been widely used in aerospace and marine navigation systems in order to provide approximations of inertial frames unaffected by the ship's movements. Humans and animals during locomotion are known to stabilize their heads to help stabilize vision and generally facilitate the measurements performed by vestibular system. In humans, the vestibular system plays a fundamental role in spatial orientation and motion planning. Similarly, in humanoid robots and other free moving robots, locating the IMU on a stabilized platform would afford key advantages that are well worth the extra complexity, for purposes of postural stabilization and motion planning, without any need for ground or environmental references.

This observations runs counter to the common practice in humanoid robot control to assume that the ground can serve as a reference for gravitational verticality. To our knowledge, head-centered coordinate frame serving as reference to topdown postural control in humanoid robots was implemented only in [24]. From this viewpoint, robot postural control from a stabilized head frame provides opportunities for implementation human-like locomotion not accessible with the current practice of humanoid motion control.

At the time of writing, we are realizing a physical prototype of a stabilized inertial platform intended to be coupled to the double inverted spatial pendulum system that we employ as a model for standing postural balance. The implementation of the techniques presented in this paper will allow us to create a control system which can stabilize itself without using any information about the environment, provided that it is placed in a gravity field.

\section{ACKNOWLEDGMENT}

The authors are indebted to Hannah Michalska for advice on the subject of nonlinear observers. I. Farkhatdinov is supported by a fellowship from Ecole Doctorale, Sciences Mecaniques, Acoustique, Electronique et Robotique de Paris. Additional funding was provided by the European Research Council, Advanced Grant PATCH, agreement No. 247300.

\section{REFERENCES}

[1] J-Y. Kim, I-W. Park, J-H. Oh, "Experimental realization of dynamic walking of the biped humanoid robot KHR-2 using zero moment point feedback and inertial measurement," Advanced Robotics, Vol. 20, No. $6,2006$.

[2] P. Veronesi, W. Werth, G. Hinterberger, M. Lackner, P. Senn, G. Weratschnig, M. Zauner, R. Mente, B. Steinwender, E. S. Petschnig, S. Preimel, "Team description paper humanoid walking machine research group," $H W M, 2007$.

[3] T. Buschmann, S. Lohmeier, H. Ulbric, "Humanoid robot Lola: design and walking control," Journal of Physiology - Paris 103 (2009), pp. 141148.

[4] D. Gouaillier, V. Hugel, P. Blazevic, C. Kilner, J. Monceaux, P. Lafourcade, B. Marnier, J. Serre, B. Maisonnier, "Mechatronic design of NAO humanoid," in Proc. of the 2009 IEEE Int. Conference on Robotics and Automation, Japan.

[5] Y. Jun, R. Ellenburg, P. Oh, "From concept to realization: designing miniature humanoids for running," J. on Systemics, Cybernetics and Informatics, Vol. 8, no. 1, 2010, pp. 8-13.

[6] Y-H. Chang, Y. Oh, D. Kim, S. Hong, "Balance control in whole body coordination framework for biped humanoid robot MAHRU-R," in Proc of the 17th IEEE Int. Symposium on Robot and Human Interactive Communication, Germany, 2008.

[7] B. J. Stephens, C. G. Atkeson "Dynamic balance force control for compliant humanoid robots," in Proceedings of the 2010 IEEE/RSJ International Conference on Intelligent Robots and Systems, Taiwan.

[8] R. A. Brooks, C. Breazeal, M. Marjanovic, B. Scassellati, M. M. Williamson, "The Cog project: building a humanoid robot," In $C$. computation for metaphors, analogy, and agents, Nehaniv (Ed.), LNCS 1562, pp. 52-87, 1999.

[9] T. Pozzo, A. Berthoz, L. Lefort, "Head stabilisation during various locomotor tasks in humans I. Normal subjects," Exp. Brain Res. 82, 1990, pp. 97-106.

[10] T. Pozzo, A. Berthoz, L. Lefort, E. Vitte, "Head stabilisation during various locomotory tasks in humans II. Patients with bilateral vestibular deficits," Exp. Brain Res. 85, 1991, pp. 208-217.

[11] T. Pozzo, Y. Levik, A. Berthoz, "Head and trunk movements in the frontal plane during complex dynamic equilibrium tasks in humans," Exp. Brain Res. 106, 1995, pp. 327-338.

[12] B. Barshan, H.F. Durrant-Whyte, "Inertial navigation systems for mobile robots," IEEE T. on Robotics and Automation, Vol. 11, No. 3, 1995.

[13] J. Leavitt, A. Sideris, J. E. Bobrow, "High bandwidth tilt measurement using low-cost sensors," IEEE/ASME T. on Mechatronics, Vol. 11, no. $3,2006$.

[14] H. Rehbinder, X. Hu, "Drift-free attitude estimation for accelerated rigid bodies," Automatica, 40, 2004, pp. 653-659.

[15] J. M. Pflimlin, T. Hamel, P. Soueres, "Nonlinear attitude and gyroscope's bias estimation for a VTOL UAV," Int. J. of Systems Science, Vol. 38, Issue 3, 2007.

[16] S. Bras, R. Cunha, J. F. Vasconcelos, C. Silvestre, P. Oliveira, "A nonlinear attitude observer based on active vision and inertial measurements," IEEE T. on Robotics, Vol. 27, Issue 4, pp. 664- 677.

[17] G. Arechavaleta, J-P. Laumond, H. Hicheur, A. Berthoz, "An optimality principle governing human walking," IEEE T. on Robotics, 24(1), pp. 5-14, 2008.

[18] D. M. Merfeld, L. Zupan, R. J. Peterka, "Humans use internal models to estimate gravity and linear acceleration," Nature, Vol. 398, 1999, pp. 615-618.

[19] D. M. Merfeld, L. H. Zupan, "Neural processing of gravitoinertial cues in humans. III. Modeling tilt and translation responses," J Neurophysiol, 87, 2002, pp. 819-833.

[20] D.E. Angelaki, K. E. Cullen, "Vestibular system: the many facets of a multimodal sense," Annual Reviews of Neuroscience. Vol. 31, pp. 125150,2008

[21] G. M. Jones, J. H. Milsum, "Frequencyresponse analysis of central vestibular unit activity resulting from rotational stimulation of the semicircular canals," J. Physiol., 219, 1971, pp. 191-215.

[22] J. Shen, A. K. Sanyal, N. A. Chaturvedi, "Dynamics and control of a 3D pendulum," in Proc. of the 43rd IEEE Conference on Decision and Control, Bahamas, 2004.

[23] P. E. Moraal, J. W. Grizzle, "Observer design for nonlinear systems with discrete-time measurements," IEEE T. on Automatic Control, Vol. 40, no. 3, 1995

[24] M. N. Sreenivasa, P. Soueres, J-P. Laumond, A. Berthoz, "Steering a humanoid robot by its head," in Proc. of the 2009 IEEE/RSJ International Conference on Intelligent Robots and Systems, pp. 4451-4456. 